\title{
Experimental Study on Mechanical Properties of Amorphous Alloy
}

\section{Fiber-reinforced Mortar}

\author{
Hongwei Ding ${ }^{1, a}$, Xiaojun Chen ${ }^{1, b}$, Titao Wang ${ }^{1, c}$, \\ Fangshu Qian ${ }^{1, d}$, Weiwei Zhang ${ }^{1, e^{*}}$ \\ 1.College of Harbour, Coastal and Offshore Engineering, Hohai University, 210098, Nanjing CHINA

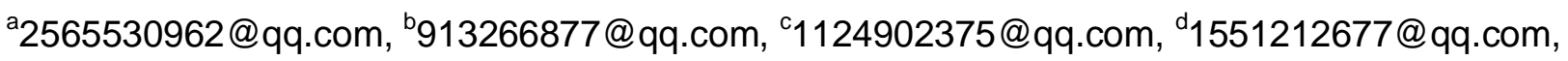 \\ em18351936505@outlook.com
}

Corresponding author: Weiwei Zhang

Keywords: amorphous alloy fibers; steel fibers; mechanical properties; cement mortar

Abstract: Compared with the steel fiber, the compressive and flexural strength of cement mortar were studied and discussed in this paper. The result showed that compared with the steel fiber, amorphous alloy fiber improved mechanical properties of cement mortar obviously. Compared with the reference specimens, with the fiber content of $1.0 \%$, the compressive and flexural strength of the amorphous alloy fiber-reinforced mortar increased by $28.6 \%$ and $51.9 \%$ separately. Compared with the steel fiber, with the fiber content of $1.0 \%$, the compressive and flexural strength of the amorphous alloy fiber-reinforced mortar increased by $9.9 \%$ and $14.5 \%$ separately. Additionally, amorphous alloy fibers dispersed evenly in the mortar, and its bonding properties is well.

\section{Introduction}

Adding fibers is an efficient way to obtain cement-based materials with high performance. Now the kinds of commonly used fiber are steel fiber, polypropylene fiber and poly acrylonitrile. However, they can't be applied to concrete structures under marine environment ${ }^{[1]}$. We must explore a new type of fiber.

Amorphous alloy fiber (AAF), or metallic glass ribbon (MGR), is a kind of ribbon amorphous materials. The atoms are topologically disordered in three-dimensional space. Without structural defects such as grain boundary and stacking fault, amorphous alloy fibers take on excellent mechanics, high resistance to corrosion and special electromagnetic performance ${ }^{[2-4]}$. Researches show that amorphous alloy fiber-reinforced concrete has good flexural properties and corrosion resistance, especially suitable for application in the port, wharf, and offshore platforms exposed chronically to the harsh marine environment.

Compared with ordinary steel fibers, this paper shows the research on compressive and flexural strength of cement mortar, adding amorphous alloy fibers. We want to make a beneficial attempt for this fiber's application in marine concrete structures.

\section{Experiment}

\section{Materials and mix proportion}

Amorphous alloy fiber and steel fiber: Amorphous alloy fiber used in this paper (specific gravity of $7.20 \mathrm{~g} / \mathrm{cm}^{3}$ ) was $2 \mathrm{~mm}$ wide, $16 \mathrm{~mm}$ long, with steel fiber in the same length. Fig. 1 gives amorphous alloy fiber's appearance and its dispersion in the mortar. It shows that amorphous alloy fibers have good toughness, good dispersion in the mortar. The properties of the fibers used in this 
study are listed in Table 1.

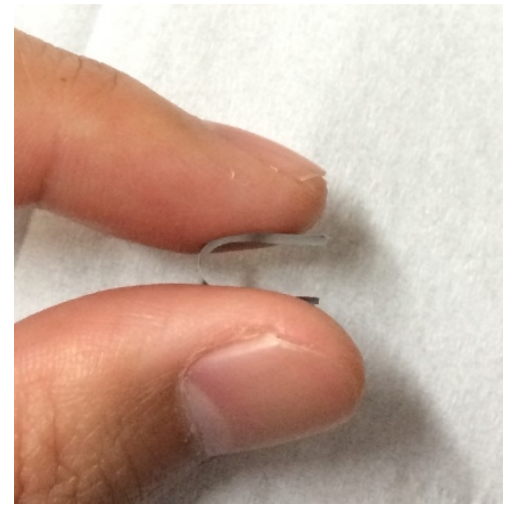

(a)

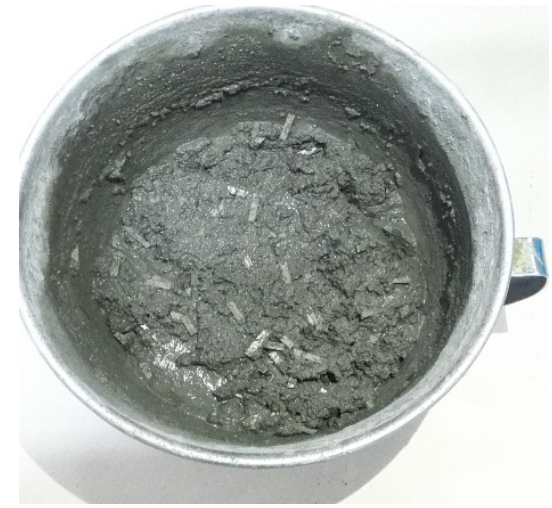

(b)

Fig.1 Amorphous alloy fiber

(a) Bending property of amorphous alloy fiber (b) Mixing of amorphous alloy fiber and mortar

Table.1 Properties of amorphous alloy fiber and straight -type steel fiber

\begin{tabular}{lll}
\hline Type & Amorphous alloy fiber & steel fiber \\
\hline Elastic modulus [MPa] & $14 \times 10^{4}$ & $20 \times 10^{4}$ \\
Specific gravity & 7.20 & 7.85 \\
Fiber length [mm] & 16 & 16 \\
Tensile strength [MPa] & 2000 & 1100 \\
Ultimate elongation /\% & & 3.5 \\
Acid/alkali resistance & High & Low \\
Electrical conductivity & High & High \\
Composition & Amorphous metal (Fe,Cr) $80 \% \quad(\mathrm{P}, \mathrm{C}, \mathrm{Si}) 20 \%$ & Alloy \\
\hline
\end{tabular}

Cement: P. C32.5 composite Portland cement producted by Conch Cement Company, Nanjing. The chemical composition of cement is listed in Table.2.

Table. 2 Chemical composition of cement

\begin{tabular}{cccccccc}
\hline $\mathrm{SiO} 2$ & $\mathrm{Al} 2 \mathrm{O} 3$ & $\mathrm{Fe} 2 \mathrm{O} 3$ & $\mathrm{CaO}$ & $\mathrm{MgO}$ & $\mathrm{SO} 3$ & Loss & $\sum$ \\
\hline 29.54 & 4.37 & 3.15 & 49.06 & 2.58 & 1.41 & 3.33 & 93.45 \\
\hline
\end{tabular}

\section{Sand: The sand is ISO standard sand.}

Mix proportion: Mortar mix proportion for $\mathrm{m}$ (cement): $\mathrm{m}$ (sand): $\mathrm{m}$ (water) was 1:3:0. 5. The fiber content were $0,0.2 \%, 0.4 \%, 0.6 \%$ and $1.0 \%$ of the volume ratio.

\section{Experimental methods}

According to certain mixing ratio, cement, sand and fibers were taken and put in JJ - 5 cement-sand blender to dryly mix evenly, then water was added to mix for 3 minutes. The mixture was put into joint testing molds of $40 \mathrm{~mm} \times 40 \mathrm{~mm} \times 160 \mathrm{~mm}$ and the models were placed on the HZJ A concrete vibration table to vibrate twice. Each time lasted one minute. Then the samples were covered with a layer of membrane and were ejected after 24 hours. In the end, standard curing was performed until the required ageing was achieved.

Tests were performed according to GB/T 17671-1999 to determine the compressive and flexural properties of fiber-reinforced mortar, with three specimens for a set. The test samples were placed in 
an EHDC300-03 compression-testing machine and a DKZ - 5000 electric bending machine separately, and the tests were performed after ageing for 7 and 28 days.

\section{Results and discussion}

\section{Compressive strength}

The experiment result of compressive tests of fiber-reinforced mortar at various fiber content is shown in Fig.2.
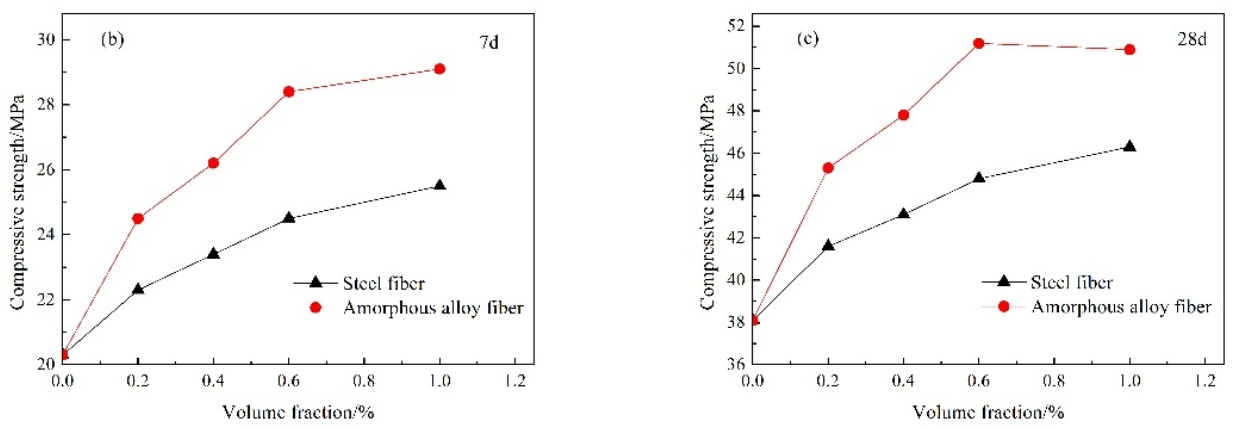

Fig.2 Compressive strength for cement mortars at various fiber content

Fig. 2 shows that compared with the reference specimens, the compressive strength increased as the cement ages and the volume fraction increased. The compressive strength of amorphous alloy fiber-reinforced mortar at volume fractions of $0.6 \%$ increased by $39.9 \%$ after ageing for 7 days, $34.4 \%$ after 28 days, in contract to the reference specimens, and increased by $15.9 \%, 14.3 \%$, respectively, compared with steel fiber-reinforced mortar at the same fiber content. At the same volume fraction, the compressive strength of amorphous alloy fiber-reinforced mortar was greater than that of steel fiber-reinforced mortar. The compressive strength after ageing for 28 days of amorphous alloy fiber-reinforced mortar at volume fractions of $0.2 \%, 0.4 \%, 0.6 \%$ and $1.0 \%$ increased by $18.9 \%, 23.3 \%, 30.4 \%$ and $28.6 \%$, respectively, compared with the reference specimens, and increased by $8.9 \%, 10.9 \%, 14.3 \%$ and $9.9 \%$, respectively, in contract to steel fiber-reinforced mortar.

When the volume fraction increased from $0.6 \%$ to $1.0 \%$, the compressive strength of amorphous alloy fiber-reinforced mortar showed a slower and slower rising trend, even decreased, while that of steel fiber-reinforced mortar continued to increase. Because amorphous alloy fibers show thin shape and the volume is small, we can't add too much. The best volume fraction is about $1.0 \%$.

\section{Flexural strength}

The experiment result of flexural tests of fiber-reinforced mortar at various fiber content is given in Fig.3.
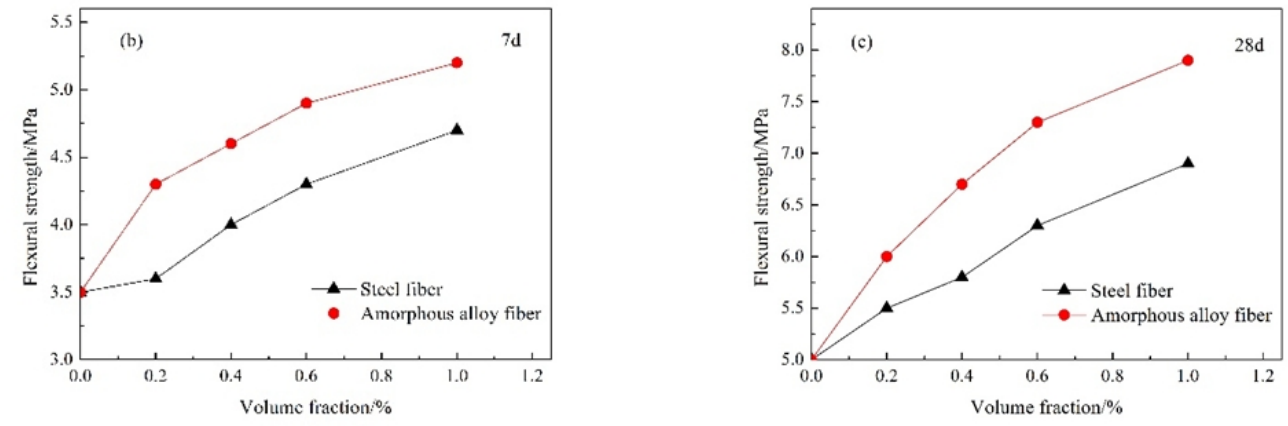

Fig.3 Flexural strength for cement mortars at various fiber content 
Fig. 3 shows that compared with the reference specimens, the flexural strength increased as the fiber content increased. The flexural strength of amorphous alloy fiber-reinforced mortar at volume fraction of $0.6 \%$ increased by $40.0 \%$ after ageing for 7 days, $46.0 \%$ after 28 days, in contract to the reference specimens, and increased by $14.0 \%, 15.9 \%$, respectively, compared with steel fiber-reinforced mortar at the same fiber content. When the fiber content of amorphous alloy fiber increased from $0.2 \%$ to $1.0 \%$, the increment of the flexural strength of amorphous alloy fiber-reinforced mortar after ageing for 28 days increased from $15.4 \%$ to $51.9 \%$. When the fiber content of steel fiber increased from $0.2 \%$ to $1.0 \%$, the increment of the flexural strength of steel fiber-reinforced mortar after ageing for 28 days increased from $3.8 \%$ to $32.7 \%$.

With the fibers in the form of needle aggregating in cement mortar, they are able to prevent the expansion of the micro cracks when cement hardens. After cracking, full load of cracking sections is applied to the cross-sectional fibers by bonding fibers and mortar. Fibers in turn spread the load on uncracking mortar matrix, so that the ultimate load of mortar becomes larger.

\section{Conclusion}

(1) Compared with the steel fibers with the same length and at the same volume fraction, amorphous alloy fibers have a larger aspect ratio, a larger root number, greater bonding properties, etc. At the same volume fraction, the number of amorphous alloy fibers is greater than that of steel fibers in unit volume of mortar. Meanwhile, amorphous alloy fibers' specific surface area is bigger. Amorphous alloy fiber can improve the compressive and flexural strength of mortar efficiently. The compressive and flexural strength of amorphous alloy fiber-reinforced mortar at volume fractions of $1.0 \%$ increased by $28.6 \%$ and $51.9 \%$ separately after ageing for 28 days, in contract to the reference specimens, and increased by $9.9 \%$ and $14.5 \%$ separately, compared with steel fiber-reinforced mortar at the same fiber content.

(2) Amorphous alloy fibers take on high tensile strength, high resistance to corrosion. Additionally, it disperses evenly in the mortar, with great bonding properties. Meanwhile, amorphous alloy fiber can improve the compressive and flexural strength of mortar efficiently at a low volume fraction. Therefore, it has a broad prospect.

\section{Acknowledgements}

This research work is funded by the Natural Science Foundation of Jiangsu Province (BK20151496), Natural Science Foundation of Shanghai Municipality (14ZR1427500) and Science and Technology research projects for Science and Technology Commission of Shanghai Municipality (13dz1202204).

\section{References:}

[1] Wu Zhongwei, Sun Wei. The new development of concrete science and technology [M]. Southeast University Press, 1995: 637-837.

[2] Song Hui, Zhu Zhenghou. The preparation and mechanical properties of FeSiB amorphous strip[J]. New technologies, 2005, (2): 47-48. (In Chinese)

[3] Yu Peng, Sun Baoan, Bai Haiyang, Wang Weihua. Exploration of plastic metal glass ribbon [J]. Physics, 2008, 37(6): 421-425. (In Chinese)

[4] Banthia N, Trottier JF, Pigeon M. Fiber pullout mechanisms. Effects of fiber geometry, loading rate and subzero temperatures. In: Proceedings of the international conference on recent developments in fiber reinforced cements and concretes Cardiff; 1989: 136-45. 